\title{
StoryGirl: Uma rede colaborativa de apoio à criança através de histórias reais programadas em Scratch
}

\author{
Beatriz Saburido Racca ${ }^{1}$ e Juliana Baptista dos Santos França ${ }^{1,2,3}$ \\ ${ }^{1}$ Departamento de Computação (DECOMP) - Universidade Federal Rural do Rio de \\ Janeiro (UFRRJ) \\ ${ }^{2}$ Programa de Pós-Graduação em Modelagem Matemática e Computacional \\ (PPGMMC) - Universidade Federal Rural do Rio de Janeiro (UFRRJ) - Seropédica, RJ, \\ Brasil \\ ${ }^{3}$ Programa de Pós-Graduação em Gestão e Estratégia (PPGE) - Universidade Federal \\ Rural do Rio de Janeiro (UFRRJ) - Seropédica, RJ, Brasil \\ \{saburido57, julibsf\}@gmail.com
}

\begin{abstract}
This paper presents the first discussions about the colaborative StoryGirl Framework. This Framework aims to create a collaborative child support network through real stories programmed in Scratch. It is part of the StoryGirl Framework discuss aspects that show the literacy of computational thinking, gender inclusion and the sharing of real experiences from child to child in an accessible language. As a result, the paper presents a pilot study on the literacy of computational thinking through stories and the initial structure of the social and collaborative child support environment.
\end{abstract}

Resumo. Este artigo apresenta as primeiras discussões sobre o Framework colaborativo StoryGirl. Este Framework visa criar uma rede colaborativa de apoio à criança através de histórias reais programadas em Scratch. Faz parte do Framework StoryGirl discutir aspectos que evidenciem a alfabetização do pensamento computacional, inclusão de gênero e o compartilhamento de experiências reais de criança para criança em uma linguagem acessivel. Como resultado, o artigo apresenta um estudo piloto sobre a alfabetização do pensamento computacional através de histórias e a estrutura inicial do ambiente social e colaborativo de apoio à criança.

\section{Introdução}

A igualdade de gênero tem sido discutida com ampla disseminação não apenas no Brasil, mas também no mundo. Segundo a ONU [ONU, 2015] a igualdade de gênero é um dos 17 objetivos a ser alcançado com a finalidade de mudar o mundo em que vivemos. De acordo com os dados do IBGE de 2013, no Brasil, a população feminina é estimada em $51 \%$, totalizando um indicativo maior de mulheres do que homens [Ribeiro et. al., 2019]. No entanto, é fato notório que as mulheres representam o quantitativo com menor valor salarial em seus cargos e menor reconhecimento profissional.

Essa mesma estatística se estende para a área de computação. Dados de 2007 mostram que apenas 24,05\% dos atuantes em computação no mercado de trabalho formal no Brasil são do gênero feminino. Em 2017 esse percentual cai ainda mais para $19,83 \%$. Esses percentuais fazem das mulheres minoria na computação e, portanto, tem motivado diferentes iniciativas para tornar essa distribuição de gênero mais homogênea nos âmbitos educacional e profissional [Softex, 2019], [Cardoso and David, 2016]. 
Com base nesta constatação, vemos a necessidade de estimular o conhecimento inerente a área de computação, atingindo principalmente crianças e jovens. Dessa maneira, espera-se que meninas e meninos cresçam entendendo que a área de tecnologia pode sim ser uma opção independente de gênero. A literatura apresenta algumas discussões sobre este espectro [Aires et. al., 2018], [Martins et al., 2019]. No entanto, esse artigo visa estimular o ensino da programação em blocos para crianças e juvenis tendo como base histórias reais vividas por essas crianças. Entende-se que o envolvimento emocional das crianças com a tecnologia trará mais elementos motivadores para a interação das crianças com esses novos recursos.

Com base nessa premissa de investigação, essa pesquisa propõem o Framework StoryGirl que visa desenvolver o pensamento computacional e habilidades interpessoais de crianças e juvenis (especialmente meninas, mas não excludente), através do desenvolvimento, construção e compartilhamento de histórias que retratam experiências vividas e de forma acessível. O pensamento computacional será estimulado através de aulas que alfabetizem as crianças e juvenis quanto a uma linguagem de programação em blocos. Esta pesquisa faz uso da linguagem de programação Scratch ${ }^{1}$ desenvolvida pelo MIT. As aulas são conduzidas presencialmente e com elementos visuais que visam atrair a atenção da criança, como desenhos e animações. Já a contação de histórias por cada criança será construída tendo por fundamento o storytelling [Lage et al. 2017].

Faz parte desta pesquisa promover o compartilhamento de histórias reais de criança para criança. Através da manipulação do Scratch elas são capazes de criar suas próprias histórias animadas e reproduzir para outros indivíduos suas experiências de forma lúdica, informal e em linguagem acessível ao universo infanto-juvenil. Na Grécia antiga, Platão dizia que todo aprendizado tem algum tipo de base emocional [Paviani 2013], seja ela positiva ou negativa. Assim, as habilidades interpessoais serão estimuladas através da criação de uma rede colaborativa de apoio segura para o compartilhamento de histórias (através dos cenários animados em scratch), onde as crianças e juvenis poderão aprender e dividir experiências sobre assuntos diversos de fundamento técnico, humano e social.

Este artigo é organizado considerando na seção 2 as referências da literatura e trabalhos relacionados sobre Web Social e redes de apoio para o estímulo do pensamento computacional. Na seção 3 é apresentado e discutido o Framework StoryGirl. Na seção 4 são discutidas algumas histórias reais e cenários animados construídos e como estas podem compor a rede colaborativa de apoio. Na seção 5 serão apresentadas as limitações e discussões dessa pesquisa que tem como pilar um projeto de extensão institucional.

\section{Aprendizagem e Redes de Apoio}

A literatura discute em algumas pesquisas o desenvolvimento do pensamento computacional com o foco em crianças [França and Tedesco, 2019]. Aono et al. (2017), Rodriguez et al. (2015), de Oliveira et al. (2019) discutem projetos cujo objetivo é o desenvolvimento do pensamento computacional em crianças através da programação de jogos em Scratch. Nessas pesquisas existe uma discussão efetiva sobre a inserção de

\footnotetext{
${ }^{1}$ https://scratch.mit.edu/
} 
competências computacionais ainda em nível educacional básico e médio na intenção de diminuir as dificuldades sofridas pelas crianças na área de exatas que é um dos responsáveis pelo abandono de alunos dos cursos de computação na universidade. Esses autores defendem que quanto mais cedo os indivíduos desenvolvem competências lógicas, mais natural se torna o processo de aprendizagem da área de exatas $\mathrm{e}$ consequentemente das áreas de tecnologia.

Outras pesquisas com foco no pensamento computacional também têm se destacado quanto ao desenvolvimento de competências acadêmicas que não necessariamente estão relacionadas ao desenvolvimento de software e jogos por crianças [França et al 2019], [Schneider et al. 2019], [Junior et al. 2019]. Há também pesquisas que discutem o desenvolvimento do pensamento computacional no público feminino, que tem sofrido com uma baixa presença na área de computação [Marquiori et al., 2019] e [Pinheiro et al. 2017]. Há ainda pesquisas que defendem o desenvolvimento do pensamento computacional através de histórias (uso da técnica storytelling), tornando o desenvolvimento dessa competência de exatas mais lúdico e divertido [Farias et al. 2019], [Gomes et al., 2017] e [de Melo Reis et al., 2017]. E outros como [Mattos et al., 2017] que defendem o ensino da programação através de jogos.

Com base nos trabalhos relacionados apresentados, observa-se que há pesquisas direcionadas ao desenvolvimento do pensamento computacional de crianças através da programação em blocos. Existe também discussões a respeito do fortalecimento feminino na área de computação, iniciando as capacitações ainda nas fases iniciais da educação formal; e ainda pesquisas que envolvem o ensino da programação através de histórias. No entanto, observa-se uma ausência de iniciativas que unam estas iniciativas e que fomentem a construção de uma rede colaborativa de apoio técnico e social às crianças. Este artigo propõem o Framework StoryGirl que tem por objetivo apoiar o desenvolvimento do pensamento computacional de crianças, além de promover um ambiente onde elas possam trocar experiencias técnicas da programação em bloco e de vida, uma vez que o produto programado por elas em Scratch serão histórias reais. Detalhes sobre o StoryGirl serão apresentados a seguir.

\section{Framework StoryGirl}
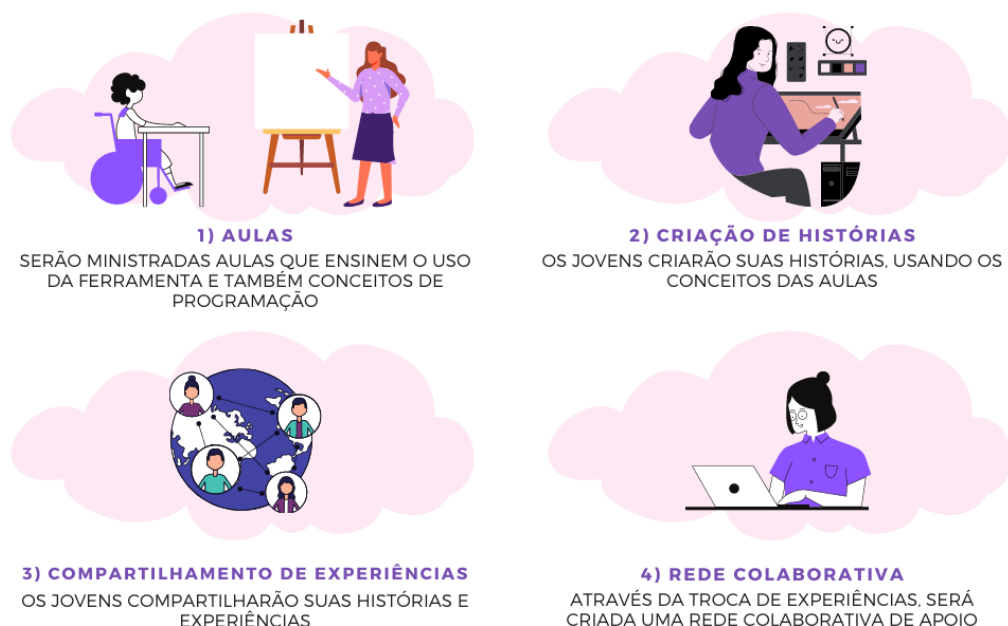

Figura 1: Framework StoryGirl 
O Framework StoryGirl é uma iniciativa originada em um projeto de extensão ainda em fase inicial em uma Universidade Federal do Brasil. Neste projeto, o principal objetivo é apresentar para crianças e juvenis (especialmente meninas, mas não exclusivo) de $4-15$ anos o universo da programação de computadores através de uma linguagem em blocos: o Scratch. Este projeto estimula o interesse das crianças para a programação através da contação de histórias (storytelling), e estas mesmas são animadas pelas crianças usando as técnicas da programação em blocos. Essas histórias podem ser de qualquer temática, tendo como único pré-requisito ser uma história real.
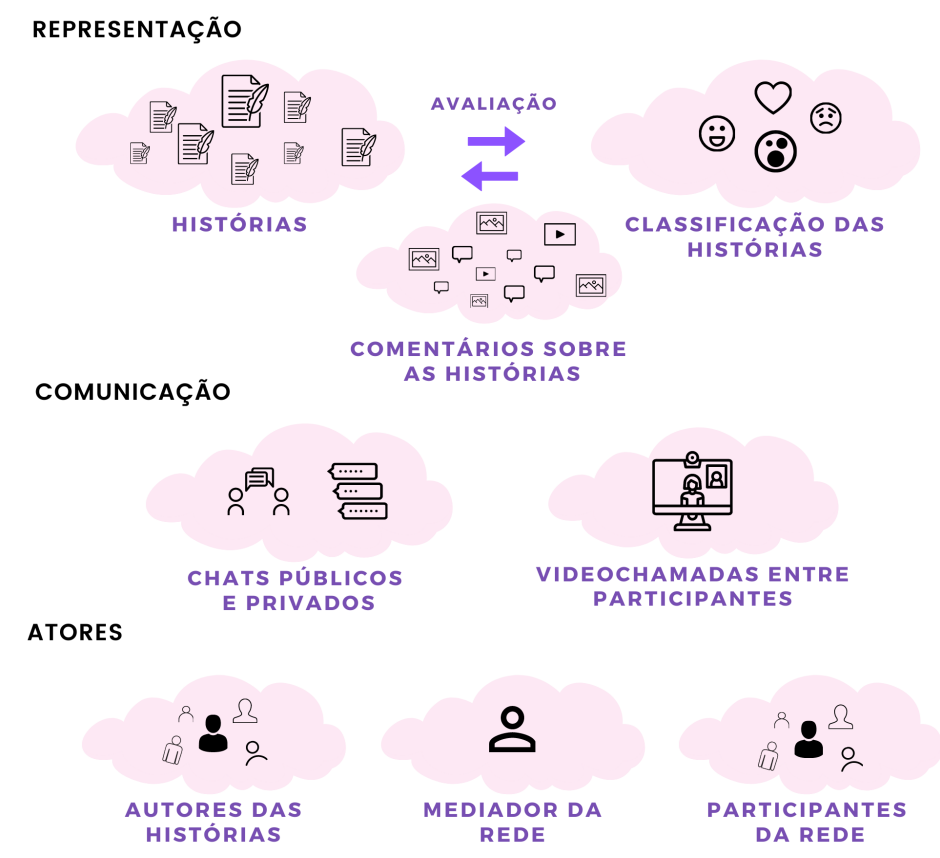

SAÍDAS
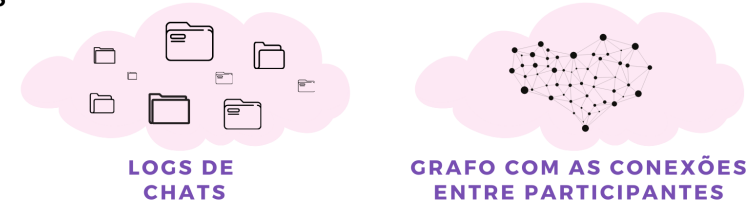

Figura 2: Estrutura do sistema de apoio ao Framework StoryGirl

Para a proposição do Framework StoryGirl ficou claro que as histórias animadas apresentam como benefício não apenas o estímulo do pensamento computacional, ou a inclusão de meninas no universo da computação, mas também o compartilhamento de experiências reais de criança para criança. Com base nisso, a Figura 1 representa as fases que compõem o Framework StoryGirl.

$\mathrm{Na}$ Fase 1 é prevista a condução de aulas cujo objetivo é informar a relação das meninas com a área de computação, apresentar o Scratch e ensinar conceitos básicos de programação em blocos. A expectativa é de 4 encontros de duas horas para a primeira fase. Na Fase 2 (2 encontros de duas horas) é esperado que as crianças e juvenis redijam suas histórias, e através dos conhecimentos adquiridos na Fase 1 sejam capazes de programar suas histórias em Scratch. A fase 2 é dividida em duas atividades: (i) redigir as histórias reais no papel ou computador; (ii) programar as histórias no Scratch. A atividade ii da Fase 2 terá como mentores as instrutoras da Fase 1 e também crianças 
participantes do projeto que se mostrarem aptas. O objetivo do projeto é incentivar as interações sociais e compartilhamento de experiências em todas as fases do Framework StoryGirl.

A Fase 3 prevê o compartilhamento das histórias animadas em uma rede segura, interação entre a turma e construção de laços afetivos entre os participantes. Esse compartilhamento se iniciará localmente onde cada criança compartilhará sua história para os demais colegas e contará sobre sua experiência na programação em bloco. A fase 3 é concluída na Fase 4, que prevê a construção de uma rede colaborativa de apoio onde as crianças, mentores e responsáveis possam trocar experiências sociais e técnicas sobre o desenvolvimento de suas competências na programação em blocos de forma global. Para a construção da rede colaborativa de apoio social, de inclusão e de desenvolvimento do pensamento computacional, é prevista a construção de um sistema web que terá como estrutura a representação da Figura 2. Nesta estrutura, observa-se o compartilhamento, avaliação e comentários das histórias pelos pares, estratégias de comunicação, construção de redes de conexões entre os participantes, e tipos de atores envolvidos no ambiente StoryGirl. Este ambiente encontra-se em fase de concepção.

\section{Piloto de Aplicação do Framework StoryGirl e Próximos Desafios}

O Framework StoryGirl encontra-se em andamento. As aulas para a Fase 1 já estão preparadas e um estudo piloto com crianças recrutadas de diferentes classes sociais, idade e nível escolar foi realizado. O objetivo deste estudo era investigar a aplicabilidade do desenvolvimento do pensamento computacional através da animação de histórias reais. Neste estudo foram relatadas seis histórias reais e três delas foram programadas em Scratch. As histórias neste estudo piloto foram programadas pelas mentoras do projeto a fim de investigar as potencialidades das histórias reais em explorar recursos básicos da programação em blocos.

A Figura 3 apresenta o cenário inicial da história de um menino de quatro anos ainda em processo de alfabetização. Já a Figura 4 e 5 apresentam o código da programação em blocos da história da Figura $3^{2}$. Observe que este é um código simples, mas efetivo na construção de um fluxo funcional onde mostra a ordenação de ações a fim de que a animação aconteça. Este código trabalha também aspectos de orientação dimensional x e y, tamanho da imagem, tempo de exposição da cena e diálogo associado.

As demais histórias animadas, retratam assuntos mais profundos como perda da avó ${ }^{3}$ e isolamento social ${ }^{4}$ de meninas de quinze e dez anos respectivamente. No entanto, os fundamentos técnicos trabalhados da programação em blocos foram basicamente os mesmos da criança de quatro anos.

\footnotetext{
${ }^{2}$ https://scratch.mit.edu/projects/412636110/

${ }^{3}$ https://scratch.mit.edu/projects/412324336

${ }^{4}$ https://scratch.mit.edu/projects/412481871/
} 


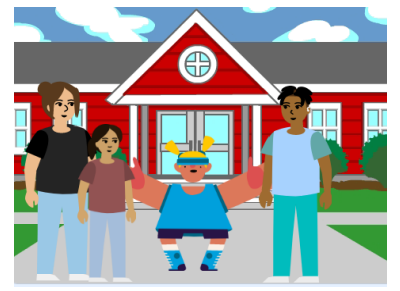

Figura 3: História do menino de 4 anos.

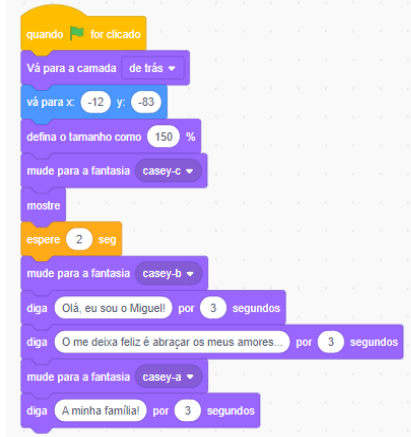

Figura 4: Código do personagem principal.

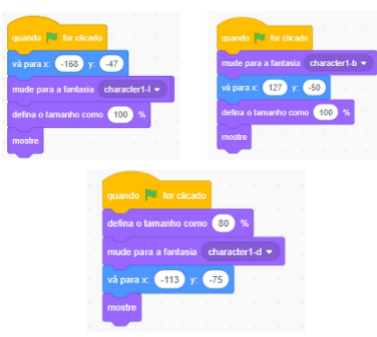

Figura 5: Código da família do personagem principal.

O próximo passo da pesquisa é conduzir as Fases 1, 2 e 3 do Framework StoryGirl em uma escola considerando alunos (especialmente meninas, mas não exclusivo) no espectro 6-15 anos, a fim de abranger crianças e juvenis que se encontrem entre o final do processo de alfabetização e o ensino médio. A intenção é investigar as dificuldades e potencialidades desses alunos em transformar suas histórias em cenários programados. Além disso, os cenários programados servirão de insumo para alimentar um ambiente tecnológico e colaborativo de troca de experiências técnicas e sociais para crianças e juvenis, habitado também por responsáveis, profissionais técnicos, de saúde e antropólogos. Em paralelo ao desenvolvimento dos cenários, o ambiente colaborativo StoryGirl (Figura 2) será desenvolvido a fim de abarcar as histórias e os demais autores envolvidos em uma atmosfera de apoio. Como estratégia de avaliação do ambiente StoryGirl, espera-se rodar alguns experimentos de uso do ambiente com os atores. Resultados gerados como log do ambiente, observação interna, questionários e entrevistas com os participantes do ambiente serão analisados e confrontados.

\section{Conclusões}

Este artigo visou discutir os primeiros passos para a concepção do Framework StoryGirl. Nele são discutidos aspectos de alfabetização do pensamento computacional em crianças e juvenis através de histórias reais, inclusão do gênero feminino no ambiente computacional e construção de uma rede colaborativa de apoio para o público infanto-juvenil, mentores e responsáveis. No artigo foi apresentado o Framework StoryGirl em 4 Fases e a estrutura inicial projetada para o ambiente colaborativo StoryGirl para apoio social e técnico de crianças foi discutida.

Como primeira investigação desta pesquisa, um projeto piloto foi conduzido com o objetivo de investigar a aplicabilidade da alfabetização de crianças e juvenis em programação de computadores através de histórias reais. Os resultados se mostraram satisfatórios e os próximos passos visam conduzir as Fases 1, 2 e 3 do Framework StoryGirl em escolas com o público infanto-juvenil. Como limitação desta pesquisa temos a indisponibilidade dos encontros presenciais nas escolas em virtude do isolamento social vivido em função da COVID-19. No entanto, é uma expectativa tornar o projeto acessível virtualmente, principalmente as fases 1 e 2 do Framework StoryGirl. Em paralelo ao desenvolvimento do projeto nas escolas, será projetado e desenvolvido funcionalmente o ambiente colaborativo StoryGirl de apoio social e técnico aos juvenis 
e crianças alfabetizadas.

\section{Agradecimentos}

Esta pesquisa recebeu apoio da FAPERJ - E-26/211.367/2019 (248406).

\section{Referências}

Aires, J., Mattos, G., Oliveira, C., Brito, A., Aragão, A. F., Alves, S., ... \& Moreira, G. (2018, July). Barreiras que impedem a opção das meninas pelas ciências exatas e computaçao: Percepçao de alunas do ensino médio. In Anais do XII Women in Information Technology. SBC.

Aono, A. H., Rody, H. V. S., Musa, D. L., Pereira, V. A., \& Almeida, J. (2017, July). A utilização do scratch como ferramenta no ensino de pensamento computacional para crianças. In Anais do XXV Workshop sobre Educação em Computação. SBC.

Cardoso, É. E. C., \& de David, T. (2016). A falta de profissionais de tecnologia de informação no mercado de trabalho. Uma Nova Pedagogia para a Sociedade Futura, 697-700.

de Melo Reis, F., Cristiano, F., Martins, D., \& da Rocha, P. (2017, October). Pensamento computacional: Uma proposta de ensino com estratégias diversificadas para crianças do ensino fundamental. In Anais do Workshop de Informática na Escola (Vol. 23, No. 1, p. 638).

de Oliveira, S. B. C., de Melo, R. F., \& de Sousa, R. P. (2019, December). Um relato de experiência da utilização do scratch no ensino de lógica de programação para crianças. In Anais da VII Escola Regional de Computação Aplicada à Saúde (pp. 169-174). SBC.

Farias, C., da Cruz, V. G., Farias, J. S., Braz, D. C., Brito, B. M., \& de Souza Carvalho, A. (2019, November). Estimulando o Pensamento Computacional: uma experiência com ScratchJr. In Anais do Workshop de Informática na Escola (Vol. 25, No. 1, p. 197).

França, R., \& Tedesco, P. (2019, November). Pensamento Computacional: Panorama dos Grupos de Pesquisa no Brasil. In Brazilian Symposium on Computers in Education (Simpósio Brasileiro de Informática na Educação-SBIE) (Vol. 30, No. 1, p. 409).

Gomes, T., Castro, F., \& Tedesco, P. C. A. R. (2017). Desenvolvendo o Pensamento Computacional na Educação Infantil: Um toolkit educacional sobre conceitos de programação baseado em storytelling transmedia. Sánchez, J, 31-40.

Junior, B., Cavalheiro, S., \& Foss, L. (2019, November). Revisitando um Jogo Educacional para desenvolver o Pensamento Computacional com Gramática de Grafos. In Brazilian Symposium on Computers in Education (Simpósio Brasileiro de Informática na Educação-SBIE) (Vol. 30, No. 1, p. 863).

Lage, B., França, J., Dias, A., Vivacqua, A., \& Borges, M. (2017, October). Aplicando o group storytelling no compartilhamento de experiências e na avaliação em sala de aula. In Anais do Workshop de Informática na Escola (Vol. 23, No. 1, p. 165). 
Marquiori, V., Oliveira, M., \& Nascimento, G. (2019, July). Letramento de Meninas em Programação através do Pensamento Computacional para Compreensão de Problemas. In Anais do XIII Women in Information Technology (pp. 109-113). SBC.

Martins, A., Silva, J., Santos, J., \& Rebouças, A. (2019, July). Fatores que Atraem e Afastam as Meninas de cursos da Área de TI. In Anais do XIII Women in Information Technology (pp. 114-118). SBC.

Mattos, M., de Araújo, L., Zucco, F., Hein, N., da Cunha, K., de Oliveira, G., ... \& Bursoni, P. (2017, July). Aplicação da prática colaborativa no desenvolvimento de um jogo para o ensino da programação. In Anais do XIV Simpósio Brasileiro de Sistemas Colaborativos (pp. 21-30). SBC.

Organização das Nações Unidas (ONU). (2015). Programa das Nações Unidas para o desenvolvimento (PNUD). Objetivos do Desenvolvimento Sustentável. Disponível em: https://nacoesunidas.org/pos2015/ods5/.

Paviani, J. (2013). Platão \& a educação. Autêntica.

Pinheiro, A., Franco, J., \& Leite, J. (2017, October). Desenvolvimento do Pensamento Computacional e discussões sobre representação feminina na Computação: um estudo de caso. In Anais dos Workshops do Congresso Brasileiro de Informática na Educaçao (Vol. 6, No. 1, p. 1111).

Ribeiro, L., Barbosa, G., Silva, I., Coutinho, F., \& Santos, N. (2019, July). Um Panorama da Atuação da Mulher na Computação. In Anais do XIII Women in Information Technology (pp. 1-10). SBC.

Rodriguez, C., Zem-Lopes, A. M., Marques, L., \& Isotani, S. (2015, October). Pensamento Computacional: transformando ideias em jogos digitais usando o Scratch. In Anais do Workshop de Informática na Escola (Vol. 21, No. 1, p. 62).

Schneider, G., Bernardini, F., \& Boscarioli, C. (2019, November). Ensino do Pensamento Computacional por meio de Internet das Coisas: Possibilidades e Desafios. In Brazilian Symposium on Computers in Education (Simpósio Brasileiro de Informática na Educação-SBIE) (Vol. 30, No. 1, p. 169).

Softex. (2019) Atuação da Mulher no Mercado de Trabalho Formal Brasileiro em Tecnologia da Informação. Arquivo eletrônico disponível em: https://www.ftp.softex.br/Inteligencia/mulheres na ti/mulheres na ti.pdf. Acessado em: julho 2020 